\title{
$\eta$ production off the proton in a Regge-plus-chiral quark approach
}

\author{
Jun $\mathrm{He} *$ \\ Institute of Modern Physics, Chinese Academy of Sciences, Lanzhou 730000, P. R. China and \\ Research Center for Hadron and CSR Physics, \\ Lanzhou University \& Institute of Modern Physics of CAS, Lanzhou 730000, China \\ B. Saghait \\ Institut de Recherche sur les lois Fondamentales de l'Univers, \\ DSM/Irfu, CEA/Saclay, 91191 Gif-sur-Yvette, France
}

(Dated: November 18, 2018)

\begin{abstract}
A chiral constituent quark model approach, embodying $s$ - and $u$-channel exchanges, complemented with a Reggeized treatment for $t$-channel is presented. A model is obtained allowing data for $\pi^{-} p \rightarrow \eta n$ and $\gamma p \rightarrow \eta p$ to be describe satisfactorily. For the latter reaction, recently released data by CLAS and CBELSA/TAPS Collaborations in the system total energy range $1.6 \lesssim W \lesssim 2.8$ $\mathrm{GeV}$ are well reproduced due to the inclusion of Reggeized trajectories instead of simple $\rho$ and $\omega$ poles. Contribution from "missing" resonances, with masses below $2 \mathrm{GeV}$, is found to be negligible in the considered processes.
\end{abstract}

PACS numbers: 13.60.Le, 12.39.Fe,12.39.Jh,14.20.Gk

* junhe@impcas.ac.cn

$\dagger$ bijan.saghai@cea.fr 


\section{INTRODUCTION}

The $\eta$ production offers an appropriate frame to study the properties of the nucleon resonances $\left(N^{*}\right)$. The zero isospin of $\eta$ is a good filter to select the produced $1 / 2$ isospin resonances compared with other meson production channels, such as $\pi N$, where isospin $3 / 2$ resonances also intervene. Among some twenty $N^{*}$ s, the lightest $S_{11}$ resonance, $N(1535)$, attracts much attention, because it is related to many interesting issues on the internal structure of nucleon resonances, such as the mixing angles [1 8 ] and multiquark components [9, 10]. The substantial branching ratio of the $N(1535) \rightarrow \eta N$ decay channel still remains to be fully understood, but it facilitates measurements of the $\eta$ production processes, given that from threshold up to roughly $200 \mathrm{MeV}$ above, the reaction mechanism is dominated by $N(1535)$. Moreover, those reactions offer the possibility of also investigating the properties of other nucleon resonances and searching for still undiscovered ones.

For half a century, hadronic and electromagnetic probes have been used for the $\eta$ production processes, essentially off the nucleon. The data for $\pi N \rightarrow \eta N$ come mainly from measurements performed in 70s [11 14] and suffer from some inconsistencies [15], except a recent experiment performed at BNL, using the Crystal Ball spectrometer [16, 17]. The published CB data [17] are high quality measurements, though limited to the close to threshold kinematics. For the photoproduction process, a healthy amount of data has been published in recent years for both differential cross section [18 21] and polarized beam asymmetry [21, 22]. Recently CBELSA/TAPS [23] and CLAS [24] Collaborations released new data with higher precision and higher energies [24].

In spite of the unbalanced data base between $\gamma N$ and $\pi N$ initiated reactions, recent theoretical efforts [25-44] are focusing, via coupled-channels approaches, on both families of processes, taking into account a significant number of intermediate and/or final states, such as $\pi N, \eta N, \rho N, \sigma N, \pi \Delta, K \Lambda, K \Sigma$. Those works are all based on effective Lagrangian approaches, where meson-baryon degrees of freedom are implemented.

Investigations based on subnucleonic degrees of freedom, via chiral constituent quark models $(\chi \mathrm{CQM})$ have been developed and successfully applied to the interpretation of photoproduction of pseudoscalar mesons on the proton, namely, $\gamma p \rightarrow \pi N$ [45], $\eta p$ [3], 46, 47], $K^{+} \Lambda$ [39], as well as the process $\pi^{-} p \rightarrow \eta n$ [48, 49]. Finally, in a recent paper a formalism in the $\chi \mathrm{CQM}$ approach [47] was extended to the $\pi^{-} p \rightarrow \eta n$ reaction and allowed performing 
a combined analysis of both channels [48].

The previous studies within the $\chi \mathrm{CQM}$ approach focus on the total-energy $(W)$ region below $2 \mathrm{GeV}$, where the best known $N^{*}$ s lie. In those formalisms contribution from $N^{*}$ s of $n>2$ shells in harmonic oscillator basis, relevant to the higher energy region, are treated as degenerate, that is, simulated by a single resonance. Recent data extending the phase space up to $W \approx 2.8 \mathrm{GeV}$ renders the degenerate approximation less reliable. One solution to that issue is to use the duality assumption [50] and to complement the $\chi$ CQM with the $t$-channel contributions, which mimic [51] the left-out higher mass resonances $(M>2$ $\mathrm{GeV}$ ). Formalisms using only Regge trajectories or associating the effective Lagrangian for $s$-channel with a Reggeized model for $t$-channel have proven to be successful in describing data above $W \approx 2 \mathrm{GeV}$ for pseudoscalar mesons: $\pi$ [52 [55], $\eta$ [43] and kaon [52, 56 60] photoproduction reactions. Besides, the MAID Group [43] showed that a Reggeized model is more appropriate than the $t$-channel exchanges described by the $\rho$ and $\omega$ poles.

So, in this work we adopt the Reggeized model to complement our $\chi$ CQM formalism [47, 48] and study the $\eta$ production, especially the very recent data released by CBELSA/TAPS [23] and CLAS [24] Collaborations.

The paper is organized as follows. In Section II, the theoretical frame of our $\chi C Q M$ approach, for both $\gamma p \rightarrow \eta p$ and $\pi^{-} p \rightarrow \eta n$, and the Reggeized model are briefly presented. The fitting procedure and numerical results for differential cross section, polarized beam asymmetry, helicity amplitudes, and partial decay widths are reported and discussed in section III. Summary and conclusion are reported in section IV.

\section{THEORETICAL FRAME}

Chiral quark model approaches, are based on the low energy QCD Lagrangian [61, 62]

$$
\mathcal{L}=\bar{\psi}\left[\gamma_{\mu}\left(i \partial^{\mu}+V^{\mu}+\gamma_{5} A^{\mu}\right)-m\right] \psi+\cdots
$$

where vector $\left(V^{\mu}\right)$ and axial $\left(A^{\mu}\right)$ currents read,

$$
V^{\mu}=\frac{1}{2}\left(\xi \partial^{\mu} \xi^{\dagger}+\xi^{\dagger} \partial^{\mu} \xi\right), A^{\mu}=\frac{1}{2 i}\left(\xi \partial^{\mu} \xi^{\dagger}-\xi^{\dagger} \partial^{\mu} \xi\right)
$$

with $\xi=\exp \left(i \phi_{m} / f_{m}\right)$ and $f_{m}$ the meson decay constant. $\psi$ and $\phi_{m}$ are the quark and meson fields, respectively. 
The amplitudes for $s$-channel resonances can then be written as,

$$
\mathcal{M}_{N^{*}}=\frac{2 M_{N^{*}}}{s-M_{N^{*}}^{2}-i M_{N^{*}} \Gamma(\mathbf{q})} e^{-\frac{\mathbf{k}^{2}+\mathbf{q}^{2}}{6 \alpha_{h o}^{2}}} \mathcal{O}_{N^{*}},
$$

where $\sqrt{s} \equiv W$ is the total energy of the system, and $\mathcal{O}_{N^{*}}$ is determined by the structure of each resonance. $\Gamma(\mathbf{q})$ in Eq. (3) is the total width of the resonance, and a function of the final state momentum q.

The transition amplitude for the $n^{\text {th }}$ harmonic-oscillator shell is

$$
\mathcal{O}_{n}=\mathcal{O}_{n}^{2}+\mathcal{O}_{n}^{3}
$$

The first (second) term represents the process in which the incoming photon and outgoing meson are absorbed and emitted by the same (different) quark [49, 62].

We use the standard multipole expansion of the CGLN amplitudes [63] to obtain the partial wave amplitudes for resonance $f_{2 I, 2 l \pm 1}$. Then the transition amplitudes for pseudoscalar meson production through photon and meson induced reactions take, respectively, the following forms:

$$
\begin{aligned}
& \mathcal{O}_{N^{*}}^{\gamma}=i f_{1 l \pm} \sigma \cdot \epsilon+f_{2 l \pm} \sigma \cdot \hat{\mathbf{q}} \sigma \cdot(\hat{\mathbf{k}} \times \epsilon)+i f_{3 l \pm} \sigma \cdot \hat{\mathbf{k}} \hat{\mathbf{q}} \cdot \epsilon+i f_{4 l \pm} \sigma \cdot \hat{\mathbf{q}} \epsilon \cdot \hat{\mathbf{q}} \\
& \mathcal{O}_{N^{*}}^{m}=f_{1 l \pm}+\sigma \cdot \hat{\mathbf{q}} \sigma \cdot \hat{\mathbf{k}} f_{2 l \pm} .
\end{aligned}
$$

We can relate the helicity amplitude for a given resonance with the multipole coefficient as in the case of photoproduction process [48]

$$
f_{l \pm}^{N^{*}}=\mp A_{l \pm}^{N^{*}}=\frac{1}{2} \epsilon\left(\frac{\Gamma_{\pi N} \Gamma_{\eta N}}{k q}\right)^{1 / 2} C_{\pi N}^{N^{*}} C_{\eta N}^{N^{*}}=\frac{1}{2 \pi(2 J+1)}\left(\frac{E_{N_{i}} E_{N_{j}}}{M_{N^{*}}^{2}}\right)^{1 / 2} A_{1 / 2}^{\pi N} A_{1 / 2}^{\eta N},
$$

where the decay width is given by

$$
\Gamma_{m}=\frac{1}{(2 J+1)} \frac{|q| E_{N}}{\pi M_{N^{*}}}\left|\frac{A_{1 / 2}^{m}}{C_{m N}^{N^{*}}}\right|^{2}
$$

and the Clebsch-Gordan coefficients are

$$
C_{m N}^{N^{*}}=\left\langle I^{N^{*}} M^{N^{*}} \mid I^{m} M^{m} I^{N} M^{N}\right\rangle,
$$

with $m \equiv \pi, \eta$.

In our approach, the photoexcitation helicity amplitudes $A_{\lambda}^{\gamma}$, as well as the strong decay amplitudes $A_{\nu}^{m}$, are related to the matrix elements of the interaction Hamiltonian [64] as 
following:

$$
\begin{aligned}
A_{\lambda}^{\gamma} & =\sqrt{\frac{2 \pi}{k}}\left\langle N^{*} ; J \lambda\left|H_{e}\right| N ; \frac{1}{2} \lambda-1\right\rangle, \\
A_{\nu}^{m} & =\left\langle N ; \frac{1}{2} \nu\left|H_{m}\right| N^{*} ; J \nu\right\rangle .
\end{aligned}
$$

For the $s$-channel, Eqs. (10) and (11) are used to determine contributions from $n \leq 2$ shells for $N^{*}$ s with $M \lesssim 2 \mathrm{GeV}$. The $u$-channel is calculated as degenerate as before [3, 47, 48, 62]. For the $t$-channel contributions, we start with Feynman diagrams for vector mesons $\rho^{0}$ and $\omega$ exchanges amplitudes. The effective Lagrangian for the vector meson exchange vertices are

$$
\begin{aligned}
& \mathcal{L}_{\gamma \eta V}=\frac{e \lambda_{V}}{m_{\eta}} \varepsilon_{\mu \nu \rho \sigma}\left(\partial^{\mu} A_{\gamma}^{\nu}\right) \phi_{\eta}\left(\partial^{\rho} V^{\sigma}\right), \\
& \mathcal{L}_{V q q}=g_{V} \bar{\psi}\left(\gamma_{\mu}+\frac{\kappa_{V} q q}{2 m_{q}} \sigma_{\mu \nu} \partial^{\nu}\right) V_{V}^{\mu} \psi,
\end{aligned}
$$

where $A_{\gamma}^{\nu}$ and $V_{V}^{\mu}$ are photon and exchanged vector mesons, respectively. Here, in line with Ref. [45] we introduced $\kappa_{V} q q$ for the constituent quark. The electromagnetic couplings of the vector mesons $\lambda_{V}$ are determined from the radiative decay widths $\Gamma_{V \rightarrow \eta \gamma}$ as MAID group [43], that is, 0.81 for $\rho$ and 0.291 for $\omega$. The values for the strong coupling constants $g_{V q q}$ and $\kappa_{V q q}$ are treated as free parameters.

With the Lagrangians in Eqs. (12) and (13), the amplitudes for $t$-channel in chiral quark model can be easily obtained as in Ref. [45]

$$
\begin{aligned}
\mathcal{M}_{V}= & e \frac{\lambda_{V} g_{V q q} e^{-(\mathbf{k}-\mathbf{q})^{2} / 6 \alpha_{h o}^{2}}}{m_{\pi}\left(t-m_{V}^{2}\right)}\left\{g _ { t } \left[1+\frac{\omega_{m}}{E_{f}+M_{f}}+\frac{\omega_{\gamma}}{E_{i}+M_{i}}\right.\right. \\
& \left.+\frac{\kappa_{V q q}}{2 m_{q}}\left(\frac{m_{\pi}^{2}}{E_{f}+M_{f}}-\left(\frac{1}{E_{f}+M_{f}}+\frac{1}{E_{i}+M_{i}}\right) k \cdot q\right)\right] \mathbf{q} \cdot\left(\mathbf{k} \times \boldsymbol{\epsilon}_{\gamma}\right) \\
& +g_{A}\left[\frac{\omega_{\gamma} \mathbf{q}^{2}}{E_{f}+M_{f}}+\frac{\omega_{m} \mathbf{k}^{2}}{E_{i}+M_{i}}-\left(\frac{\omega_{\gamma}}{E_{i}+M_{i}}+\frac{\omega_{m}}{E_{f}+M_{f}}\right) \mathbf{q} \cdot \mathbf{k}\right. \\
& +\frac{\kappa_{V q q}}{2 m_{q}}\left(\omega_{m} \mathbf{k}^{2}+\omega_{\gamma} \mathbf{q}^{2}+\frac{\omega_{\gamma} \omega_{m}}{E_{f}+M_{f}}+\frac{\left(\omega_{\gamma} \omega_{m}-m_{\pi}^{2}\right)}{E_{i}+M_{i}} \mathbf{k}^{2}\right. \\
& \left.\left.-\left(\omega_{\gamma}+\omega_{m}+\frac{\omega_{m}^{2}}{E_{f}+M_{f}}+\frac{\omega_{\gamma}^{2}}{E_{i}+M_{i}}-\frac{k \cdot q}{E_{i}+M_{i}}+\frac{\mathbf{q} \cdot \mathbf{k}}{E_{f}+M_{f}}\right) \mathbf{q} \cdot \mathbf{k}\right)\right] i \boldsymbol{\sigma} \cdot \boldsymbol{\epsilon}_{\gamma} \\
& +g_{A}\left[\frac{\omega_{m}}{E_{f}+M_{f}}+\frac{\kappa_{V q q}}{2 m_{q}}\left(\omega_{m}+\frac{\omega_{m}^{2}}{E_{f}+M_{f}}-\frac{k \cdot q}{E_{f}+M_{f}}+\frac{\mathbf{k} \cdot \mathbf{q}}{E_{i}+M_{i}}\right)\right] i \boldsymbol{\sigma} \cdot \mathbf{k q} \cdot \boldsymbol{\epsilon}_{\gamma} \\
& \left.-g_{A}\left[\frac{\omega_{\gamma}}{E_{f}+M_{f}}+\frac{\kappa_{V q q}}{2 m_{q}}\left(\omega_{\gamma}+\frac{\mathbf{k}^{2}}{E_{i}+M_{i}}+\frac{\omega_{\gamma} \omega_{m}}{E_{f}+M_{f}}\right)\right] i \boldsymbol{\sigma} \cdot \mathbf{q q} \cdot \boldsymbol{\epsilon}_{\gamma}\right\}
\end{aligned}
$$


where $k \cdot q=\omega_{\gamma} \omega_{m}-\mathbf{k} \cdot \mathbf{q}$ is the four-momentum product. As in Ref. [62], a Lorentz boost is adopted here, which was introduced to inlude the relativistic effect in the calcualtion of the proton form factor [65]. The exponential term $e^{-(\mathbf{k}-\mathbf{q})^{2} / 6 \alpha_{h o}^{2}}$ comes from the nucleon wave function, which plays the role of a form factor. However, the MAID Group [43] study showed that the form factor is not required for the Reggeized model. Hence, we remove the exponential term in our calculations (see [IIB). $g_{A}$ is the axial vector coupling and is defined in the quark model as $\left\langle N_{f}\left|\sum_{j} \hat{I}_{j}^{v} \boldsymbol{\sigma}_{j}\right| N_{i}\right\rangle \equiv g_{A}\left\langle N_{f}|\boldsymbol{\sigma}| N_{i}\right\rangle$, where $\hat{I}_{j}^{v}$ is the isospin operator for the exchanged vector meson. The factor $g_{t}$ comes from the isospin space, $g_{t} \equiv\left\langle N_{f}\left|\sum_{j} \hat{I}_{j}^{v}\right| N_{i}\right\rangle$.

In the Reggeized model the main change is substituting the meson exchange poles by the Regge propagator:

$$
\frac{1}{t-m_{V}^{2}} \rightarrow \mathcal{P}_{\text {Regge }}^{V}=\left(\frac{s}{s_{0}}\right)^{\alpha_{V}(t)-1} \frac{\pi \alpha_{V}^{\prime}}{\sin \left[\pi \alpha_{V}(t)\right]} \frac{\mathcal{S}_{V}+e^{-i \pi \alpha_{V}(t)}}{2} \frac{1}{\Gamma\left(\alpha_{V}(t)\right)},
$$

where $s_{0}=1 \mathrm{GeV}^{2}$ is the reference mass scale and $\mathcal{S}_{V}= \pm 1$ is the trajectory's signature. The gamma function $\Gamma\left(\alpha_{V}(t)\right)$ suppresses poles of the propagator in the unphysical region. The vector-meson Regge trajectory is taken in the following linear form

$$
\alpha_{V}(t)=\alpha_{V}^{\circ}+\alpha_{V}^{\prime} t
$$

with $t$ the Mandelstam variable, and read for $\rho$ and $\omega$, respectively, as

$$
\begin{aligned}
& \alpha_{\rho}(t)=0.55+0.8 t \\
& \alpha_{\omega}(t)=0.44+0.9 t
\end{aligned}
$$

\section{RESULTS AND DISCUSSION}

\section{A. Fitting procedure}

Using the CERN MINUIT code, we have fitted simultaneously the following data sets and PDG values:

\section{- Spectrum of known resonances:}

Known resonances: We use as input the PDG values [66] for masses and widths, for which the uncertainties are handled as in Ref. [47]. The 12 known nucleon resonances, with $M \lesssim 2 \mathrm{GeV}$, considered in this work are: 
$\boldsymbol{n}=1: S_{11}(1535), S_{11}(1650), D_{13}(1520), D_{13}(1700)$, and $D_{15}(1675) ;$

$\boldsymbol{n = 2 :} P_{11}(1440), P_{11}(1710), P_{13}(1720), P_{13}(1900), F_{15}(1680), F_{15}(2000)$, and $F_{17}(1990)$.

Besides the above isospin- $1 / 2$ resonances, we fit also the mass of the $\Delta(1232)$ resonance. However, spin-3/2 resonances do not intervene in the $\eta$ photoproduction.

\section{- Observables for $\gamma p \rightarrow \eta p$ :}

Differential cross-section: Data base includes 3349 data points for $1.5 \lesssim W \lesssim 2.8$ GeV, coming from the following labs: MAMI [67], CLAS [18], ELSA [19], LNS [20], and GRAAL [21]. Only statistical uncertainties are used. For the most recent data from CBELSA/TAPS [23] and CLAS [24] Collaborations, both statistical and systematic uncertainties are considered to avoid too strong constraints due to their very small statistical uncertainties.

Polarization observables: For polarized beam asymmetry $\Sigma, 184$ data points for $1.5 \lesssim W \leq 1.9 \mathrm{GeV}$ from GRAAL [21] and ELSA [22] are used with statistical uncertainties.

The target asymmetry $(T)$ data [68] are not included in our data base. Actually, those 50 data points bear too large uncertainties to put significant constraints on the parameters [47].

- Observables for $\pi^{-} p \rightarrow \eta n$ : In line with Ref. [41], the used data base includes 354 differential cross sections, for $1.5 \lesssim W \leq 2.0 \mathrm{GeV}$, coming from: Deinet [11], Richards [12], Debenham [13], Brown [14], Prakhov [17]. Uncertainties are treated as in Ref. [41]

In summary, 3887 experimental values are fitted. To do so, we have a total of 19 free parameters, not all of them adjusted on all the data sets, as explained below.

In Table \ we summarize the list of adjustable parameters and their extracted values. Note that the reported uncertainties are those produced by the MINUIT code and should be considered as lower limits.

Two of the parameters, namely, the non-strange quarks average mass $\left(m_{q}\right)$ and the harmonic oscillator strength $\left(\alpha_{h o}\right)$ are involved in fitting both mass spectrum and $\eta$-production data. The QCD coupling constant $\left(\alpha_{s}\right)$ and the confinement constants $(\Omega$ and $\Delta)$, intervene 
TABLE I. Adjustable parameters with their extracted values, where $m_{q}, \alpha_{h o}, \Omega, \Delta, M$, and $\Gamma$ are in $\mathrm{MeV}$.

\begin{tabular}{|c|c|c|c|}
\hline & Parameter & Ref. [48] & Present work \\
\hline & $m_{q}$ & 312 & $310 \pm 5$ \\
\hline & $\alpha_{h o}$ & 348 & $309 \pm 2$ \\
\hline & $\alpha_{s}$ & 1.96 & $1.60 \pm 0.02$ \\
\hline & $\Omega$ & 437 & $421 \pm 4$ \\
\hline & $\Delta$ & 460 & $460 \pm 1$ \\
\hline & $g_{\eta N N}$ & 0.376 & $0.276 \pm 0.005$ \\
\hline \multirow[t]{2}{*}{$P_{13}(1720):$} & $C_{P_{13}(1720)}^{\gamma}$ & 0.37 & $0.22 \pm 0.01$ \\
\hline & $C_{P_{13}(1720)}^{\pi}$ & -0.89 & $-0.85 \pm 0.03$ \\
\hline \multirow[t]{3}{*}{ New $S_{11}$ : } & $M^{\gamma}$ & 1715 & $1700 \pm 1$ \\
\hline & $\Gamma^{\gamma}$ & 207 & $473 \pm 10$ \\
\hline & $C_{N^{*}}^{\gamma}$ & 0.51 & $1.18 \pm 0.03$ \\
\hline \multirow[t]{2}{*}{$N(1535):$} & $M^{\gamma}$ & -- & $1532 \pm 1$ \\
\hline & $\Gamma^{\gamma}$ & -- & $140 \pm 1$ \\
\hline \multirow[t]{2}{*}{$u$-channel: } & $C_{u}^{\gamma}$ & -- & $0.71 \pm 0.03$ \\
\hline & $C_{u}^{\pi}$ & -- & $1.39 \pm 0.05$ \\
\hline \multirow[t]{4}{*}{ t-channel } & $g_{\rho q q}$ & -- & $1.90 \pm 0.22$ \\
\hline & $\kappa_{\rho q q}$ & -- & $-0.20 \pm 0.01$ \\
\hline & $g_{\omega q q}$ & -- & $4.88 \pm 0.16$ \\
\hline & $\kappa_{\omega q q}$ & -- & $-0.26 \pm 0.02$ \\
\hline
\end{tabular}

only in fitting the $\eta$-production data via the configuration mixing mechanism. In Table【the extracted values within the present work are given and compared to those reported in our previous paper [48] where a single higher mass resonance was considered, corresponding to a degenerate treatment of $n>2$ shells nucleon resonances, instead of the $t$-channel mechanism. The quark mass and the harmonic oscillator strength $\left(\alpha_{h o}\right)$ are close to the values in the previous fitting [48] while the QCD coupling constant $\left(\alpha_{s}\right)$ gets decreased by about $17 \%$. For the other parameters, the extracted values come out close to those used by Isgur-Karl [69] and Capstick-Roberts [70]: $E_{0}=1150 \mathrm{MeV}, \Omega \approx 440 \mathrm{MeV}$, and $\Delta \approx 440 \mathrm{MeV}$. 
The remaining parameters are involved in the fitting of $\eta$-production data. With respect to the $\eta$-nucleon coupling constant $g_{\eta N N}$, our result favors a rather small coupling around $g_{\eta N N}=0.3$, which is compatible with those deduced from fitting only the $\eta$ photoproduction [3, 71]. Comparable values for that coupling are also reported in Refs. [72 75].

As already mentioned, the $S_{11}(1535)$ is the dominant resonance in the $\eta$ production processes up to $W \lesssim 1.7 \mathrm{GeV}$. Hence we treat its Breit-Wigner mass and width as free parameters. Both extracted values come out within the PDG ranges: $M=1535 \pm 10 \mathrm{MeV}$ and $\Gamma=150 \pm 25 \mathrm{MeV}$.

Two strength parameters are introduced for the resonance $P_{13}(1720)$, in line with Ref. [47], and treated as adjustable in order to avoid its otherwise too large contribution resulting from direct calculation. The value of that parameter extracted from the photoproduction reaction, is close to that obtained [47] by fitting data below $W \lesssim 2 \mathrm{GeV}$.

In our previous works [47, 48], three new resonances, $S_{11}, D_{13}$, and $D_{15}$, were introduced, in line with findings by several authors [3, 30, 31, 36, 39, 43, ,76 82], with extracted masses roughly between 1.7 and $2.1 \mathrm{GeV}$. The new $D_{13}$ state was found to be negligible in the $\eta$ photoproduction [47, 48] and, hence, is not considered here. The contribution from $D_{15}$ $(M>2 \mathrm{GeV})$ might overlap with the $t$-channel contributions in this work. Hence we have removed it to avoid double-counting problem. The third $S_{11}$ is still kept in this work and its extracted mass comes out close to the previous works [3, 47, 48] while the width is about 470 $\mathrm{MeV}$, which is larger than the value in Ref. [47]. Such a large width might be an indication of additional $S_{11}$ resonance(s), as reported in Ref. [36]. Finally, for the process $\pi^{-} p \rightarrow \eta n$, we deal only with the known resonances, as in Refs. [41, 48].

Now we discuss the $u$ - and $t$-channels treatments.

In chiral quark model approaches, the $u$-channel contribution is handled as degenerate [62]. In the work of MAID group [43], it was suggested that the $u$-channel contributions may be important in reproducing the behavior of differential cross section at extreme backward angles. Hence we introduce a unique global adjustable parameter for this channel. Numerical results show about $30 \%$ deviations from unity, expected within exact $S U(6) \otimes O(3)$ symmetry. 
Below, we give the expressions relating the meson-nucleon-nucleon couplings to those at the quark level:

$$
\begin{aligned}
g_{t}^{V} g_{V q q} & =g_{V N N}, \\
g_{A}^{V} \frac{g_{V q q}}{m_{q}}\left(1+\kappa_{V q q}\right) & =\frac{g_{V N N}}{m_{N}}\left(1+\kappa_{V N N}\right) .
\end{aligned}
$$

Values for $g_{V q q}$ and $\kappa_{V q q}$, with $V \equiv \rho, \omega$, have been extracted by fitting the photoproduction data, as reported in Table【. For the $t$-channel small values of $\kappa_{V q q}$ are found as expected. For the remaining parameters we use the quark model values [45] : $g_{A}^{\omega}=1, g_{t}^{\omega}=3, g_{A}^{\rho^{\circ}}=5 / 3$ and $q_{t}^{\rho^{\circ}}=1$. Those values are comparable with the values reported by other authors [38, 43 , 83 85], within the uncertainties on extracted values for $g^{A}$ and $g^{t}$ in constituent quark model.

Using our extracted values for $V q q$ vertices couplings (Table I. last four rows) and Eqs. (19) and (20) lead, for $V N N$ vertices, to results reported in Table III and compared with results from three other works. Our values for $\rho$ are smaller than those extracted by the MAID analysis [43] and also coming from Bonn [86] and Nijmegen [87] potentials. Note that results from these latter potentials differ between themselves by $30 \%$ to $50 \%$. For $\omega$ case our value for $g_{\omega N N}$ comes out larger than the MAID analysis [43], but stands in-between those produced by nucleon-nucleon potentials. The $\kappa_{\omega N N}$ deviates significantly from vanishing values of potentials. Detailed discussion on the $V N N$ couplings values can be found in Refs. [27, 28].

TABLE II. Vector-meson nucleon-nucleon couplings.

\begin{tabular}{lcccc}
\hline \hline Ref. & $g_{\rho N N}$ & $\kappa_{\rho N N}$ & $g_{\omega N N}$ & $\kappa_{\omega N N}$ \\
\hline Present work & 1.90 & 3.0 & 14.6 & -0.25 \\
MAID [43] & 2.4 & 3.7 & 9.0 & 0 \\
Bonn potential [86] & 3.34 & 6.1 & 15.8 & 0 \\
Nijmegen potential [87] & 2.76 & 4.2 & 11.1 & 0.02 \\
\hline \hline
\end{tabular}

At this stage, having presented various parameters of our model, either fitted or taken from literature, we proceed to rather detailed discussion, per data set, of the quality of our fit.

In Table III, rows 2 to 7 give the total $\chi^{2}$ (column 4) and the $\chi^{2}$ per data point (column 6) for $\gamma p \rightarrow \eta p$ differential cross sections. The $\chi_{d p}^{2}$ comes out smaller than 2 for data published 
between years 1995 and 2007, by collaborations from MAMI [67] (MAMI95), LNS [20] (LNS06) and GRAAL [21] (GRAAL07). Those data span a total energy range going from threshold to $W \lesssim 1.9 \mathrm{GeV}$.

TABLE III. $\chi^{2}$ for the $\gamma p \rightarrow \eta p$ differential cross section (rows 2 to 8 ) and polarized beam asymmetry (rows 9 and 10); differential cross section of the reaction $\pi^{-} p \rightarrow \eta n$ (rows 11 and 15); and mass spectrum of isospin $1 / 2$ baryon resonances (row 16).

\begin{tabular}{|c|c|c|c|c|c|}
\hline Observable & Collaboration/author & $\mathrm{W}(\mathrm{GeV})$ & $\sum \chi^{2}$ & $N_{d p}$ & $\sum \chi_{d p}^{2}$ \\
\hline \multirow[t]{7}{*}{$\frac{d \sigma}{d \Omega}(\gamma p \rightarrow \eta p)$} & MAMI94 [67] & $1.49-1.54$ & 183.73 & 100 & 1.84 \\
\hline & CLAS02 [18] & $1.53-2.12$ & 929.49 & 190 & 4.90 \\
\hline & CLAS09 [24] & $1.68-2.80$ & 2595.48 & 1081 & 2.40 \\
\hline & ELSA05 [19] & $1.53-2.51$ & 1250.66 & 631 & 1.98 \\
\hline & ELSA09 [23] & $1.59-2.37$ & 2028.88 & 680 & 2.98 \\
\hline & LNS06 [20] & $1.49-1.74$ & 313.16 & 180 & 1.74 \\
\hline & GRAAL07 [21] & $1.49-1.91$ & 629.13 & 487 & 1.29 \\
\hline \multirow[t]{2}{*}{$\Sigma(\gamma p \rightarrow \eta p)$} & ELSA07 [22] & $1.57-1.84$ & 42.21 & 34 & 1.24 \\
\hline & GRAAL07 [21] & $1.50-1.91$ & 883.60 & 150 & 5.89 \\
\hline \multirow[t]{5}{*}{$\frac{d \sigma}{d \Omega}\left(\pi^{-} p \rightarrow \eta n\right)$} & Prakhov et al. [17] & $1.49-1.52$ & 39.45 & 84 & 0.47 \\
\hline & Deiinet et al. [11] & $1.51-1.70$ & 127.01 & 80 & 1.59 \\
\hline & Richards et al. [12] & $1.51-1.90$ & 122.31 & 64 & 1.91 \\
\hline & Debenham et al. [13] & $1.49-1.67$ & 16.71 & 24 & 0.70 \\
\hline & Brown et al. [14] & $1.51-2.45$ & 159.82 & 102 & 1.57 \\
\hline$N^{*}$ Spectrum & PDG [66] & & 51.2 & 15 & 3.42 \\
\hline Total & & & 9372.2 & 3902 & 2.40 \\
\hline
\end{tabular}

The best reduced $\chi^{2}$ is obtained for GRAAL07 measurements, with close to 500 data points. The worse $\chi_{d p}^{2}$ is given by the first results published [18] in 2002 by the CLAS Collaboration (CLAS02), which show discrepancies with the most recent results [23, 24], including those by the same Collaboration (CLAS09), which extends the higher limit in energy from $W \approx 2.1$ to $2.8 \mathrm{GeV}$ and embodies almost 6 times more data points. Measurements performed at ELSA show different trends, namely, the early work [19] of that collaboration published in 2005 (ELSA05), goes slightly higher in energy than their latest results [23], 
but shows a smaller $\chi_{d p}^{2}$ by about $30 \%$, with comparable number of data points. We will come back to these considerations in the next Sec. Results for polarized beam asymmetry are reported in Table III, rows 8 and 9. We get an excellent agreement with the ELSA measurements [22] (ELSA07), but a large $\chi_{d p}^{2}$ for GRAAL07 data [21].

In summary, with respect to the photoproduction observables the $\chi_{d p}^{2}$ turns out to be around 2.5, with significant discrepancies within various data sets and/or observables.

Finally, in rows 9 to 13 in Table III, our results for the strong channel are given. The overall $\chi_{d p}^{2}$ is 1.3 , though it shows significant variations according to the data set, but still it stays below 2 .

We will come back to these considerations in the next Sec.

To end this section, we present our results for resonances spectrum and roles played by those resonances in the reaction mechanisms of the processes considered in this work.



FIG. 1. (Color online) The spectrum of baryon resonances from PDG [66] (green bands) and from the present work for known (red bans), missing (yellow bands), and new (white band) resonances. The black bands indicate the variations of $\chi^{2}$ after turning off the corresponding resonance within our full model. 
In Fig. 1 are depicted mass values from PDG isospin $1 / 2$ resonances with $M \lesssim 2 \mathrm{GeV}$, our results for the 12 known $N^{*}$ s listed in Sec. IIIA, OGE generated missing resonances [47] ( $P_{11}$ (1899), $P_{13}$ (1942), $P_{13}(1965), P_{13}(2047)$, and $F_{15}$ (1943)) and the introduced third $S_{11}$. The masses generated by our formalism compare well enough with the PDG values in line with other CQM approaches [69, 88]. The same observation is valid for missing resonances, as discussed in our previous work [47]. The new $S_{11}$ has no counterpart within known, neither missing resonances.

In order to investigate the importance of the 18 resonances of our approach, we have switched off each of them one by one. The five missing resonances show no significant effects in line with our previous findings [47]. For the other $N^{*}$ s, the black part in each bar (Fig. 1) indicates the relative change in $\chi^{2}$ with that specific resonance turned off. Among the 12 known $N^{*}$ s, the most significant ones, with decreasing importance, are: $S_{11}(1535)$, $S_{11}(1650), D_{13}(1520), F_{15}(1680), P_{13}(1900)$. Finally, the new $S_{11}(1700)$ appears to be the second most important ingredient of our model.

In the following we move to the observables for the $\gamma p \rightarrow \eta p$ and $\pi^{-} p \rightarrow \eta n$ processes, and compare our results with the data. To get better insights into the reaction mechanism, we also report on the contributions from resonances which have significant effects on $\chi^{2}$ for both processes studied here.

\section{B. Observables for $\gamma p \rightarrow \eta p$}

In Fig. 2 differential cross section results are depicted at twenty four energies going from close to threshold, $E_{\gamma}^{l a b}=0.715 \mathrm{GeV}(W=1.49 \mathrm{GeV})$ up to $E_{\gamma}^{l a b}=3.70 \mathrm{GeV}(W=2.80 \mathrm{GeV})$. At each energy three curves are compared with the data: a) full model, b) contribution from solely $S_{11}(1535)$, c) contributions from the Reggeized $t$-channel. Note that, while full model embodies $u$-channel, its contribution is too small to be shown in the Figure.

Comparing our model (full curves) with various data shows that the general agreement is acceptable and there is no anomalous behavior in the whole phase space. Discrepancies within those data, in some cases with more than $2 \sigma$, make clear problems that have to be faced in fitting such a data base, which is reflected in the $\chi_{d p}^{2}=2.5$. The dotted curves show contributions due only to $S_{11}(1535)$, which has a dominant role near to threshold and up to $W \approx 1.7 \mathrm{GeV}$, where $t$-channel effects already start becoming visible. Note that 


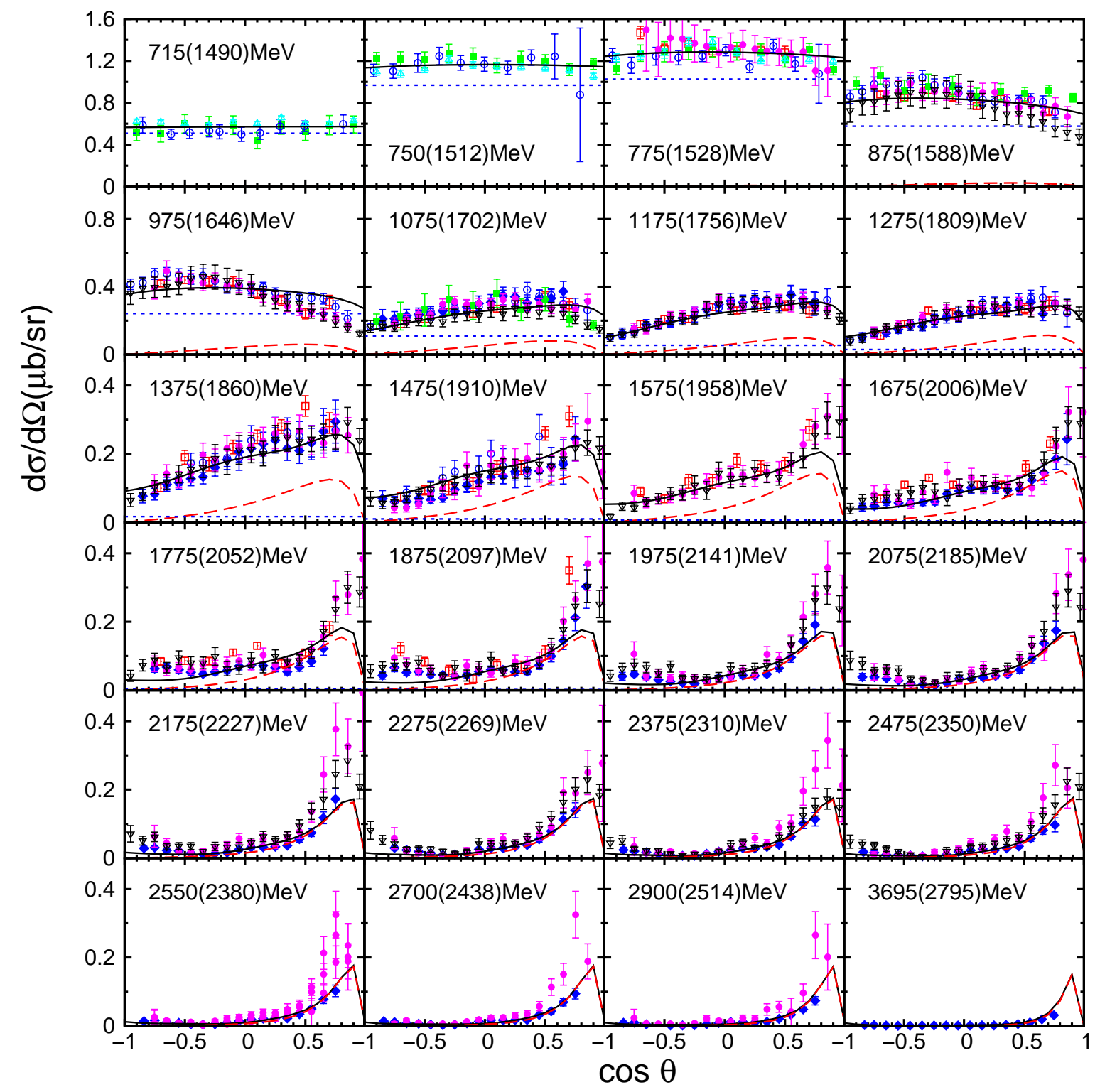

FIG. 2. (Color online) Differential cross section for $\gamma p \rightarrow \eta p$ as a function of $\cos \theta_{\eta}$ for various values of photon energy in the lab frame. The values in parenthesis are the corresponding total energy of the system $W$. The curves are: full model (full), $S_{11}(1535)$ (dotted), and $t$-channel (Dashed). Data are from CLAS02 (open squares) 18], CLAS09 (filled diamonds) 24], LNS (filled squares) 20], GRAAL (open circles) 21], ELSA05 (filled circles) 19], ELSA09 (down triangles) 23] and MAMI (open triangles) [67].

the dashed curves correspond to contributions exclusively from $t$-channel, without further minimizations. From $W \approx 2 \mathrm{GeV}$ on, this latter channel gains more and more importance with increasing energy and completely dominates the model results above $W \approx 2.1 \mathrm{GeV}$.

In the range $2.2 \lesssim W \lesssim 2.3 \mathrm{GeV}$ the extreme angles are not very well reproduced, 
indicating very likely that higher mass $N^{*}$ s are needed and/or a more extended treatment of the $u$-channel contributions is desirable. In our work, $u$-channel contributions turn out to be very small in the whole energy region, with a maximum contribution of roughly $8 \%$ around $W \approx 1.65 \mathrm{GeV}$ and almost vanishing above $W \approx 1.9 \mathrm{GeV}$.

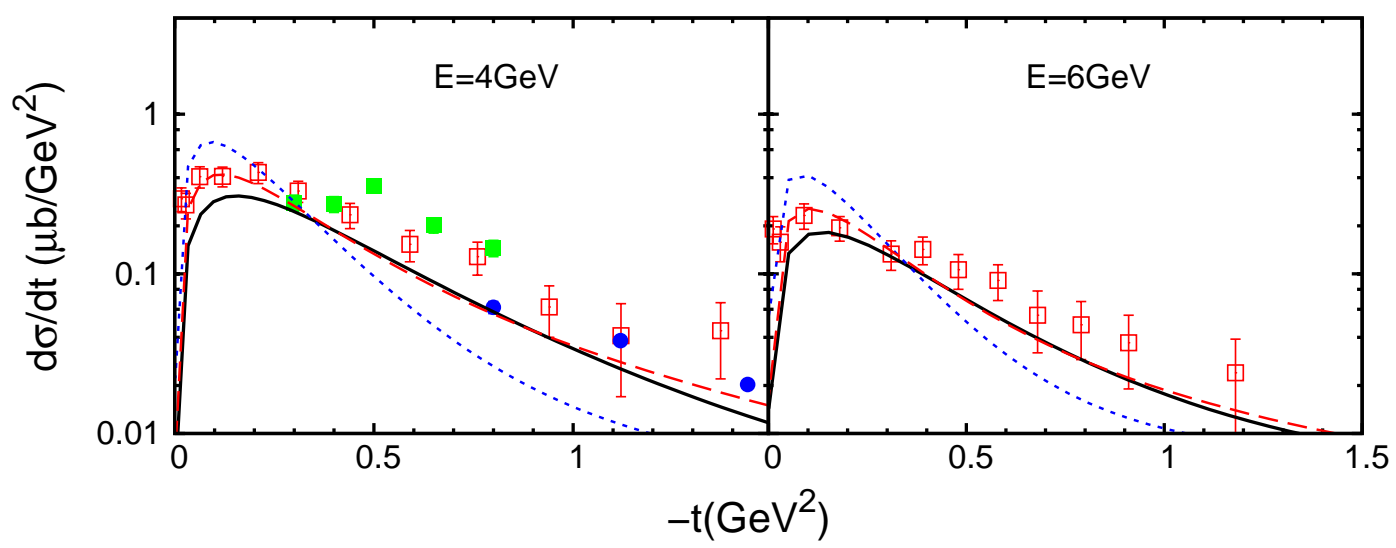

FIG. 3. (Color online) Differential cross section for $\gamma p \rightarrow \eta p$ as a function of $t$ at 4 and $6 \mathrm{GeV}$ of photon energy in the lab frame. The solid curves are our full model. The dotted curves are obtained by keeping the expotential form factor (Eq. [14]), the dashed curves are for $s_{0}=3 \mathrm{GeV}^{2}$ with the expontential form factor kept. Data are from Ref. [89] (empty square), Ref. [90] (filled square) and Ref. 24] (fiiled circles).

Here, we would like to comment on our Regge treatment. The canonical approach is to fix the Reggeized model parameters by fitting data at energies above the resonance region [52, 53, 57, 91 93]. In this work, given that recent data cover both the resonance region and above, though not higher than $3.7 \mathrm{GeV}$, we have used only the recent data for consistency considerations. However, we report the predictions of our model at 4 and $6 \mathrm{GeV}$ (Fig. 3, full curves) and find reasonable agreement with the differential cross section data at high energies [89, 90]. From there, and the fact that data above $\approx 2 \mathrm{GeV}$ show clearly the dominace of $t$-channel (Fig. 2, dashed curves), we infer that the Regge approach parmeters extracted within the present wok, by fitting the recent data, are reliable enough. In Fig. 3 we show also the effects of the expotential form factor in Eq. 14, that we removed. By adding that term to our full model, as expected we get a significant damping effects (dotted curves), which can be compensated by increasing the mass scale $s_{0}$ from 1 to $3 \mathrm{GeV}^{2}$ (dashed curves).

To give a complete picture of the $\eta$ photoproduction, polarized beam asymmetries are presented in Fig. 4. The full model (full curves) describes the data satisfactorily, except at 




FIG. 4. (Color online) Same as Fig. 2] but for polarized beam asymmetry for $\vec{\gamma} p \rightarrow \eta p$. Data are from GRAAL (open circle) 21] and ELSA (filled circle) [22].

$W=1.688$ and $1.907 \mathrm{GeV}$, which, given the small uncertainties on GRAAL data, illustrates the large $\chi^{2}$ obtained for that data set. Here, the two other curves have following ingredients: dotted curves come from the full model, with $S_{11}(1535)$ switched off, and the dashed curves are obtained by turning off $t$-channel contributions. These two sets of results are also obtained without further minimizations.

As in the case of the differential cross section, the beam asymmetry is dominated by $S_{11}(1535)$ (dotted curves), up to $W \lesssim 1.7 \mathrm{GeV}$. Above that energy, $t$-channel contributions become visible, with effects comparable to differential cross section case in the same energy range.

\section{Observables for $\pi^{-} p \rightarrow \eta n$}

In Fig. 5 we report our results for the full model (full curves), contributions due to only $S_{11}(1535)$ (dotted curves) and those exclusively from $u$-channel.

As already mentioned the data base for $\pi^{-} p \rightarrow \eta n$ suffers from serious inconsistencies, especially with increasing energy. With this in mind, we limit ourselves to the $W \lesssim 2 \mathrm{GeV}$ region.

The overall agreement between theory and heterogeneous data is acceptable. The well known dominance of $S_{11}(1535)$ shows up at the lowest energies. For $W \geq 1.6 \mathrm{GeV} u$-channel 


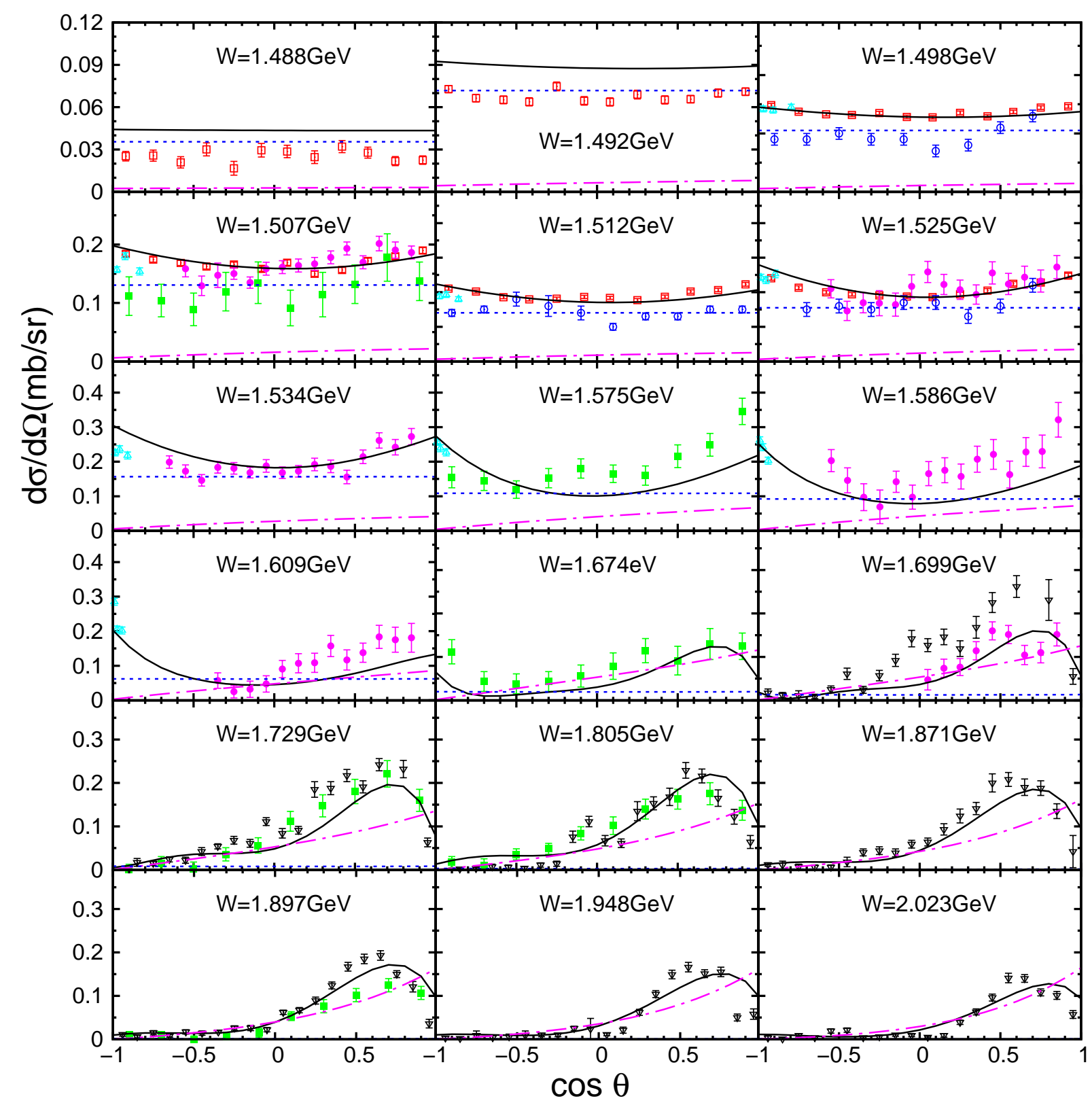

FIG. 5. (Color online) Differential cross section for $\pi^{-} p \rightarrow \eta n$ as a function of $\cos \theta_{\eta}$ for various values of $W$. The curves are: full model (full curves), $S_{11}(1535)$ (dotted), $u$-channel (dash-dotted). Data are from Prakhov et al. (open squares) [17], Richards et al. (filled squares) 12], Morrison et al. (open circles) [16], Deinet et al. (filled circles) 11], Debenham et al. (open triangles) [13], Brown et al. (down triangles) 14].

brings in significant contributions. However, neither of these two ingredients reproduces the shape of the measured cross sections. So here, the reaction mechanism again embodies destructive interferences among $N^{*}$ s contributions.

The main novelty here, compared to our previous work [48] without $t$-channel, appears at 
most backward angles, where the present model reproduces those data points much better.

\section{Helicity amplitudes}

In Table IV] we present the predictions of our full model for the helicity amplitudes and the partial decay widths of $N^{*} \rightarrow \eta N, \pi N$ decay channels, for all $n=1$ and 2 shell resonances generated by the quark model, and reported in PDG.

TABLE IV. Helicity amplitudes and decay widths for resonances, with $\Gamma_{\eta(\pi) N}^{P D G}=\Gamma_{t o t} \cdot B r_{\eta(\pi) N}$ in PDG 66]. Here $\sigma$ is the sign for $\pi N \rightarrow \eta N$ as in Ref. [94].

\begin{tabular}{l|rlrr|rr|rr}
\hline \hline Resonances & $A_{1 / 2}$ & $A_{1 / 2}^{P D G}$ & $A_{3 / 2}$ & $A_{3 / 2}^{P D G}$ & $\sigma \sqrt{\Gamma_{\eta N}}$ & $(\sigma) \sqrt{\Gamma_{\eta N}^{P D G}}$ & $\sqrt{\Gamma_{\pi N}}$ & $\sqrt{\Gamma_{\pi N}^{P D G}}$ \\
\hline$S_{11}(1535)$ & 64 & $90 \pm 30$ & & & 7.38 & $8.87_{-1.37}^{+1.37}$ & 8.90 & $8.22_{-1.60}^{+1.59}$ \\
$S_{11}(1650)$ & 58 & $53 \pm 16$ & & & -2.40 & $1.95_{-1.57}^{+0.94}$ & 12.09 & $11.31_{-1.98}^{+1.95}$ \\
$P_{11}(1440)$ & -26 & $-65 \pm 4$ & & & 1.62 & & 19.38 & $13.96_{-3.48}^{+4.41}$ \\
$P_{11}(1710)$ & -54 & $9 \pm 22$ & & & -1.02 & $2.49_{-0.88}^{+1.75}$ & 3.19 & $3.87_{-1.64}^{+3.20}$ \\
$P_{11}(2100)$ & 3 & & & & -1.01 & & 5.77 & $5.34_{-2.16}^{+2.16}$ \\
$P_{13}(1720)$ & 166 & $18 \pm 30$ & -63 & $-19 \pm 20$ & 2.44 & $2.83_{-0.71}^{+1.04}$ & 24.35 & $5.48_{-1.60}^{+2.27}$ \\
$P_{13}(1900)$ & 29 & & 3 & & -1.19 & $8.35_{-2.20}^{+2.11}$ & 13.11 & $11.38_{-2.21}^{+2.20}$ \\
$D_{13}(1520)$ & -17 & $-24 \pm 9$ & 142 & $166 \pm 5$ & 0.33 & $0.51_{-0.08}^{+0.07}$ & 14.26 & $8.31_{-0.89}^{+0.71}$ \\
$D_{13}(1700)$ & -5 & $-18 \pm 13$ & 2 & $-2 \pm 24$ & -0.61 & $0.00_{-0.00}^{+1.22}$ & 4.90 & $3.16_{-1.58}^{+1.58}$ \\
$D_{15}(1675)$ & -6 & $19 \pm 8$ & -9 & $15 \pm 9$ & -1.88 & $0.00_{-0.00}^{+1.28}$ & 7.56 & $7.75_{-1.00}^{+0.87}$ \\
$F_{15}(1680)$ & 13 & $-15 \pm 6$ & 123 & $133 \pm 12$ & 0.43 & $0.00_{-0.00}^{+1.18}$ & 13.61 & $9.37_{-0.54}^{+0.53}$ \\
$F_{15}(2000)$ & -1 & & 11 & & -0.36 & & 3.71 & $4.00_{-2.18}^{+6.20}$ \\
$F_{17}(1990)$ & 5 & $30 \pm 29$ & 7 & $86 \pm 60$ & -1.18 & $0.00_{-0.00}^{+2.17}$ & 7.03 & $4.58_{-1.55}^{+1.55}$ \\
\hline \hline
\end{tabular}

Our results (Table IV) come out in line with those from other similar approaches (see Tables I and II in Ref. [70]). Among the adjustable parameters given in Table【, only the first five intervene in the entries discussed here. Those parameters are close enough to the values obtained without $t$-channel contribution in our previous work [47]. So, results reported in Table IV] are not very different from those in Table IV of Ref. [47]. Nevertheless the present results show a general tendency to decrease the helicity amplitudes with respect to the previous results. This feature brings the predicted values closer to those reported in PDG. The 
most significant improvements concern $S_{11}(1650)$ and $D_{13}(1520)$, though the latter has a rather marginal role, except in producing the right curvature of the photoproduction polarization observables. The partial decay widths $N^{*} \rightarrow \pi N$ also mostly follow a decreasing behavior, leading to significant improvements for $S_{11}(1535)$ and $S_{11}(1650)$. With respect to the five missing resonances $\left(P_{11}(1899), P_{13}(1942), P_{13}(1965), P_{13}(2047), F_{15}(1943)\right.$, not

shown in Table IV, the helicity amplitudes differ slightly from those in Ref. [47], and braching ratios show more sensitivity to the t-channel treatments, but variations still remain below $10 \%$. Finally, none of the results display a drastic undesirable change due to the inclusion of $t$-channel contributions.

\section{SUMMARY AND CONCLUSION}

Chiral constituent quark approach, embodying $n \leq 2$ shell resonances, has proven to be an appropriate formalism in interpreting data for processes $\gamma p \rightarrow \eta p$ and $\pi^{-} p \rightarrow \eta n$ from threshold up to $W \lesssim 2 \mathrm{GeV}$. The effective Lagrangian approaches, based on mesonnucleon degrees of freedom, allow also comparable success with respect to the available data. However, the $\chi C Q M$ offers on the one hand insights into the subnucleonic structure of hadrons, and on the other requires (much) smaller number of adjustable parameters, a number which increases significantly within effective Lagrangian treatments while including an increasing number of nucleon resonances.

Developing a formalism adequate for energies above $W \approx 2 \mathrm{GeV}$ implies, either the extension of the $\chi C Q M$ formalism to $n>2$ shells, or the inclusion of $t$-channel contributions. In the present work we have adopted the second option and complemented our $\chi C Q M$ formalism with a Reggeized trajectories treatment. Such an effort is motivated by the new data released by the CBELSA/TAPS [23] and CLAS [24] collaborations. The s-channel contributions are calculated in the quark level starting from the effective chiral Lagrangian and $u$-channel is treated as degenerate. For the $t$-channel a Reggeized model is introduced at quark level.

A database, with about 4000 data points, for both $\eta$ photoproduction and $\pi^{-} p \rightarrow \eta n$ processes was used in the fitting procedure, with 19 adjustable parameters. The whole database is well reproduced with an average of reduced $\chi^{2}$ of about 2.4 , except for the CLAS results [18] published in 2002. As an outcome, we reach the following conclusions: 
- The reaction mechanism in $s$-channel is dominated by five known resonances, namely, $S_{11}(1535), S_{11}(1650), D_{13}(1520), F_{15}(1680)$, and $P_{13}(1900)$, and one new $S_{11}$ resonance with $M \approx 1.7 \mathrm{GeV}$. The missing resonances generated by the OGE mechanism show no influence on the studied reactions mechanisms.

- The Reggeized $t$-channel is indispensable in reproducing data above $W \approx 2 \mathrm{GeV}$.

- The $u$-channel contributions, though treated in degenerate approximation, play significant role in extreme forward angles for $d \sigma / d \Omega\left(\pi^{-} p \rightarrow \eta n\right)$.

- The main coupling constant, $g_{\eta N N}$ turns out to be rather small, with a value of $\approx 0.3$.

- The extracted couplings for the $\rho$ and $\omega$ mesons come out compatible with values known from other sources.

- The mass and width of the main nucleon resonance, $S_{11}$ (1535), left as free parameters, get values in agreement with those reported in PDG. This result emphasizes the stability of the minimization procedure.

To go further in such investigations, there is a real need for new $\pi N \rightarrow \eta N$ data in the energy range covered already by photoproduction data, corresponding to the whole range of the nucleon resonance masses. From theoretical side, extending the $\chi C Q M$, with $s$-channel embodying resonances above $n=2$ shell seems desirable and a work in that direction is in progress. That approach allows including all known resonances, even those with masses higher than $2 \mathrm{GeV}$. Then that work is expected to provide a suitable context to test the duality principle within our formalism.

\section{ACKNOWLEDGEMENTS}

This project is supported by National Natural Science Foundation of China under Grants 10905077, the Project-sponsored by SRF for ROCS, SEM under grant HGJO90402, and the special Foundation of president fellowship of Chinese Academy of sciences under grant YZ080425.

[1] N. Isgur and G. Karl, Phys. Lett. B72, 109 (1977). 
[2] N. Isgur, Phys. Rev. D62, 054026 (2000).

[3] B. Saghai and Z. Li, Eur. Phy. J. A11, 217 (2001).

[4] J. Chizma and G. Karl, Phys. Rev. D68, 054007 (2003).

[5] J. He and Y.-B. Dong, Nucl. Phys. A725, 201 (2003).

[6] J. He and Y.-B. Dong, J. Phys. G29, 2737 (2003).

[7] S. Capstick and W. Roberts, Fizika B13, 271 (2004).

[8] B. Saghai and Z. Li, Few Body Syst. 47, 105 (2010).

[9] C. S. An and B. S. Zou, Eur. Phys. J. A39, 195 (2009).

[10] C.-S. An and B.-S. Zou, Sci. Sin. G52, 1452 (2009).

[11] W. Deinet et al., Nucl. Phys. B11, 495 (1969).

[12] W. B. Richards et al., Phys. Rev. D 1, 10 (1970).

[13] N. C. Debenham et al., Phys. Rev. D12, 2545 (1975).

[14] R. M. Brown et al., Nucl. Phys. B153, 89 (1979).

[15] M. Clajus and B. M. K. Nefkens, PiN Newslett. 7, 76 (1992).

[16] T. W. Morrison, Ph.D. thesis, The George Washington University (1999).

[17] S. Prakhov et al., Phys. Rev. C72, 015203 (2005).

[18] M. B. Dugger et al. (CLAS), Phys. Rev. Lett. 89, 222002 (2002).

[19] V. Crede et al. (CB-ELSA Collaboration), Phys. Rev. Lett. 94, 012004 (pages 5) (2005).

[20] T. Nakabayashi et al., Phys. Rev. C74, 035202 (pages 7) (2006).

[21] O. Bartalini et al. (The GRAAL), Eur. Phys. J. A33, 169 (2007).

[22] D. Elsner et al. (CBELSA), Eur. Phys. J. A33, 147 (2007).

[23] V. Crede et al. (CBELSA/TAPS), Phys. Rev. C80, 055202 (2009).

[24] M. Williams et al. (CLAS), Phys. Rev. C80, 045213 (2009).

[25] T. Feuster and U. Mosel, Phys. Rev. C58, 457 (1998).

[26] T. Feuster and U. Mosel, Phys. Rev. C59, 460 (1999).

[27] G. Penner, Ph.D. thesis, Universität Giessen (2002).

[28] G. Penner and U. Mosel, Phys. Rev. C66, 055211 (2002).

[29] A. V. Anisovich et al., Eur. Phys. J. A25, 427 (2005).

[30] A. V. Sarantsev, V. A. Nikonov, A. V. Anisovich, E. Klempt, and U. Thoma, Eur. Phys. J. A25, 441 (2005).

[31] M. Batinic et al. (1997), nucl-th/9703023. 
[32] S. Ceci, A. Svarc, and B. Zauner, Phys. Rev. Lett. 97, 062002 (2006).

[33] R. A. Arndt, W. J. Briscoe, I. I. Strakovsky, and R. L. Workman, Int. J. Mod. Phys. A22, 349 (2007).

[34] T. P. Vrana, S. A. Dytman, and T. S. H. Lee, Phys. Rept. 328, 181 (2000).

[35] A. Matsuyama, T. Sato, and T. S. H. Lee, Phys. Rept. 439, 193 (2007).

[36] G.-Y. Chen, S. Kamalov, S. N. Yang, D. Drechsel, and L. Tiator, Nucl. Phys. A723, 447 (2003).

[37] D. Y. Chen, Y. B. Dong, M. M. Giannini, and E. Santopinto, Nucl. Phys. A782, 62 (2007).

[38] A. Gasparyan, J. Haidenbauer, C. Hanhart, and J. Speth, Phys. Rev C68, 045207 (2003).

[39] B. Julia-Diaz, B. Saghai, T.-S. Lee, and F. Tabakin, Phys.Rev. C73, 055204 (2006).

[40] B. Julia-Diaz, T. S. H. Lee, A. Matsuyama, and T. Sato, Phys. Rev. C76, 065201 (2007).

[41] J. Durand, B. Julia-Diaz, T. S. H. Lee, B. Saghai, and T. Sato, Phys. Rev. C78, 025204 (2008).

[42] J. Durand, B. Julia-Diaz, T. S. H. Lee, B. Saghai, and T. Sato, Int. J. Mod. Phys. A24, 553 (2009).

[43] W.-T. Chiang, S. N. Yang, L. Tiator, M. Vanderhaeghen, and D. Drechsel, Phys. Rev. C68, $045202(2003)$.

[44] W.-T. Chiang, B. Saghai, F. Tabakin, and T. S. H. Lee, Phys. Rev. C69, 065208 (2004).

[45] Q. Zhao, J. S. Al-Khalili, Z. P. Li, and R. L. Workman, Phys. Rev. C65, 065204 (2002).

[46] J. He, B. Saghai, Z. Li, Q. Zhao, and J. Durand, Eur. Phys. J. A35, 321 (2008).

[47] J. He, B. Saghai, and Z. Li, Phys. Rev. C78, 035204 (2008).

[48] J. He and B. Saghai, Phys. Rev. C80, 015207 (2009).

[49] X.-H. Zhong, Q. Zhao, J. He, and B. Saghai, Phys. Rev. C76, 065205 (2007).

[50] P. D. B. Collins, An Introduction to Regge Theory and High-Energy Physics (1997), Cambridge University Press.

[51] B. Saghai and F. Tabakin, Phys. Rev. C53, 66 (1996).

[52] M. Guidal, J. M. Laget, and M. Vanderhaeghen, Nucl. Phys. A627, 645 (1997).

[53] A. Sibirtsev et al., Eur. Phys. J. A34, 49 (2007).

[54] A. Sibirtsev, J. Haidenbauer, S. Krewald, U. G. Meissner, and A. W. Thomas, Eur. Phys. J. A41, 71 (2009). 
[55] A. Sibirtsev, J. Haidenbauer, F. Huang, S. Krewald, and U. G. Meissner, Eur. Phys. J. A40, $65(2009)$.

[56] M. Guidal, J. M. Laget, and M. Vanderhaeghen, Phys. Rev. C68, 058201 (2003).

[57] T. Corthals, J. Ryckebusch, and T. Van Cauteren, Phys. Rev. C73, 045207 (2006).

[58] T. Corthals, D. G. Ireland, T. Van Cauteren, and J. Ryckebusch, Phys. Rev. C75, 045204 (2007).

[59] P. Vancraeyveld, L. De Cruz, J. Ryckebusch, and T. Van Cauteren, Phys. Lett. B681, 428 (2009).

[60] P. Vancraeyveld, L. De Cruz, J. Ryckebusch, and T. Van Cauteren, EPJ Web Conf. 3, 03013 (2010).

[61] A. Manohar and H. Georgi, Nucl. Phys. B234, 189 (1984).

[62] Z.-p. Li, H.-x. Ye, and M.-h. Lu, Phys. Rev. C56, 1099 (1997).

[63] G. F. Chew, M. L. Goldberger, F. E. Low, and Y. Nambu, Phys. Rev. 106, 1345 (1957).

[64] L. A. Copley, G. Karl, and E. Obryk, Nucl. Phys. B13, 303 (1969).

[65] A. L. Licht and A. Pagnamenta, Phys. Rev. D2, 1150 (1970).

[66] C. Amsler et al. (Particle Data Group), Phys. Lett. B667, 1 (2008).

[67] B. Krusche et al., Phys. Rev. Lett. 74, 3736 (1995).

[68] A. Bock et al., Phys. Rev. Lett. 81, 534 (1998).

[69] N. Isgur and G. Karl, Phys. Rev. D19, 2653 (1979).

[70] S. Capstick and W. Roberts, Prog. Part. Nucl. Phys. 45, S241 (2000).

[71] Z.-p. Li and B. Saghai, Nucl. Phys. A644, 345 (1998).

[72] L. Tiator, C. Bennhold, and S. S. Kamalov, Nucl. Phys. A580, 455 (1994).

[73] M. Kirchbach and L. Tiator, Nucl. Phys. A604, 385 (1996).

[74] S.-L. Zhu, Phys. Rev. C61, 065205 (2000).

[75] V. G. J. Stoks and T. A. Rijken, Phys. Rev. C59, 3009 (1999).

[76] Z.-p. Li and R. Workman, Phys. Rev. C53, 549 (1996).

[77] M. M. Giannini, E. Santopinto, and A. Vassallo, Eur. Phys. J. A12, 447 (2001).

[78] M. Ablikim et al. (BES), Phys. Rev. Lett. 97, 062001 (2006).

[79] S.-s. Fang, Int. J. Mod. Phys. A21, 839 (2006).

[80] V. A. Tryasuchev, Eur. Phys. J. A22, 97 (2004).

[81] T. Mart, A. Sulaksono, and C. Bennhold (2004), nucl-th/0411035. 
[82] N. G. Kelkar and B. K. Jain, Nucl. Phys. A612, 457 (1997).

[83] D. Drechsel, O. Hanstein, S. S. Kamalov, and L. Tiator, Nucl. Phys. A645, 145 (1999).

[84] R. M. Davidson, N. C. Mukhopadhyay, and R. S. Wittman, Phys. Rev. D43, 71 (1991).

[85] O. Dumbrajs et al., Nucl. Phys. B216, 277 (1983).

[86] R. Machleidt, K. Holinde, and C. Elster, Phys. Rept. 149, 1 (1987).

[87] T. A. Rijken, Phys. Rev. C73, 044007 (2006).

[88] N. Isgur and G. Karl, Phys. Rev. D18, 4187 (1978).

[89] W. Braunschweig et al., Phys. Lett. B33, 236 (1970).

[90] J. Dewire et al., Phys. Lett. B37, 326 (1971).

[91] J. M. Laget, Phys. Rev. C72, 022202 (2005).

[92] T. E. Rodrigues et al., Phys. Rev. Lett. 101, 012301 (2008).

[93] A. Sibirtsev, J. Haidenbauer, S. Krewald, and U. G. Meissner, Eur. Phys. J. A44, 169 (2010).

[94] R. Koniuk and N. Isgur, Phys. Rev. D21, 1868 (1980). 